\title{
MIR376B Pre-miRNA
}

National Cancer Institute

\section{Source}

National Cancer Institute. MIR376B Pre-miRNA. NCI Thesaurus. Code C82828.

MIR376B is an oligoribonucleotide that is encoded by the human MIR376B gene and has a role in the regulation of gene expression. 